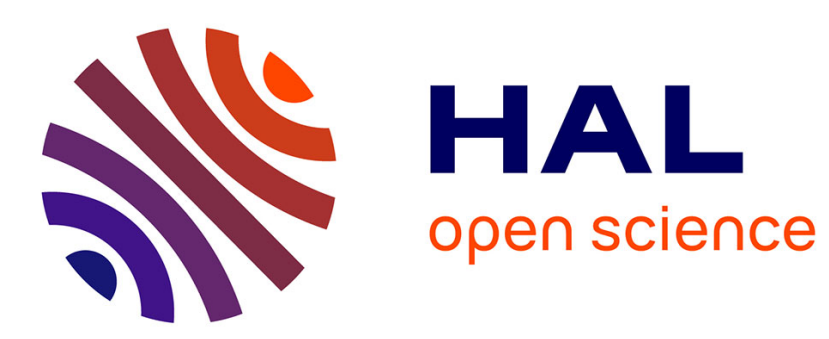

\title{
Kolm et le démembrement de la propriété de soi. Une justification "libérale" de la redistribution des revenus
}

Jean-Sébastien Gharbi

\section{To cite this version:}

Jean-Sébastien Gharbi. Kolm et le démembrement de la propriété de soi. Une justification "libérale" de la redistribution des revenus. Revue de Philosophie Economique / Review of Economic Philosophy, 2015, 16 (2), pp.107-144. hal-02015995

\section{HAL Id: hal-02015995 \\ https://hal.science/hal-02015995}

Submitted on 12 Feb 2019

HAL is a multi-disciplinary open access archive for the deposit and dissemination of scientific research documents, whether they are published or not. The documents may come from teaching and research institutions in France or abroad, or from public or private research centers.
L'archive ouverte pluridisciplinaire HAL, est destinée au dépôt et à la diffusion de documents scientifiques de niveau recherche, publiés ou non, émanant des établissements d'enseignement et de recherche français ou étrangers, des laboratoires publics ou privés. 


\title{
Kolm et le démembrement de la propriété de soi Une justification "libérale" de la redistribution des revenus
}

\author{
Jean-Sébastien Gharbi ${ }^{1}$
}

\begin{abstract}
Résumé : Le concept de propriété de soi occupe une place tout à fait centrale dans les débats sur les fondements philosophiques des théories libérale de la justice sociale. Le but de cet article est de mettre en évidence l'intérêt, l'originalité et la portée de la position qu'adopte Serge-Christophe Kolm (2005) vis-à-vis de ce concept. Le démembrement de la propriété de soi est, en effet, l'une de ses propositions majeures, tant par elle-même, que par les conséquences qu'elle a en termes de redistribution des revenus et d'égalisation des libertés individuelles de choix.
\end{abstract}

Mots clés : Redistribution des revenus, Propriété de soi, libéralisme, ELIE, Kolm.

\section{Kolm and the Dismemberment of Self-Ownership A "Liberal" Argument in favor of Income Redistribution}

\begin{abstract}
The concept of self-ownership is central in debates on philosophical groundings of liberal theories of social justice. This article discusses the relevance, the originality, and the scope of Serge-Christophe Kolm's original position (2005) regarding this key concept. Indeed, dismemberment of self-ownership is one of Kolm's major proposals, which has far-reaching consequences in terms of income redistribution, and of individual freedoms of choice equalization.
\end{abstract}

Keywords: Income redistribution, Self-Ownership, Liberalism, ELIE, Kolm.

Classification JEL: D 31, H 20, P 26.

1. Regards, Université de Reims Champagne-Ardenne. Contact : jsgharbi@gmail.com.

Des versions antérieures de ce texte ont nettement bénéficié de remarques et commentaires de Claude Gamel, Philippe Grill, Serge-Christophe Kolm, André Lapied, Jean Magnan de Bornier, Cléa Sambuc, Yves Meinard et Alain Wolfelsperger, ainsi que de deux rapporteurs anonymes. L'auteur reste bien évidemment seul responsable des défauts qui subsistent.

$\mathrm{Ce}$ travail a bénéficié d'un financement par l'European Union Seventh Framework Programme (FP7/2007-2013), programme LIBEAC, sous le numéro d'agrément n ${ }^{\circ}$ PIRSES-GA-2012-317767. 


\section{Introduction}

Le concept de propriété de soi se définit classiquement, et depuis le Second Traité du gouvernement de John Locke ${ }^{2}(1690$, p. 22), comme le droit de contrôle de l'usage de sa propre personne. Plus précisément, on peut le décomposer en trois propositions : 1) Je suis propriétaire de mon corps - affirmation qui signifie simplement que, dans la mesure où je ne serais pas libre si l'on pouvait utiliser mon corps sans mon consentement, je suis (et dois, par principe, être) le seul à avoir le droit d'en disposer. 2) Je suis propriétaire de mon travail puisque je suis propriétaire de mon corps, je suis libre de contrôler l'utilisation de son énergie et de ses talents. 3) Je suis propriétaire des fruits de mon travail - puisque je suis propriétaire de mon travail, les conséquences directes de celui-ci me reviennent de droit. On voit aisément que cette façon de présenter le rapport à soi-même en termes de propriété et de considérer que les trois propositions ci-dessus sont logiquement indissociables peut être utilisée comme argument contre l'idée d'une redistribution des revenus issus du travail. C'est la position qu'adoptent les auteurs qu'il est aujourd'hui convenu d'appeler «libertariens de droite » (Nozick, Rothbard, D. Friedman).

Comme tous les égalitaristes libéraux (Sen, Cohen, Arneson, Rawls), Kolm refuse l'idée que les individus sont pleinement propriétaires d'eux-mêmes. Cohen (1995), sans doute la tentative la plus développée d'un égalitariste libéral pour discuter cette idée dans le détail, tentait de justifier ce refus sans y parvenir de façon tout à fait satisfaisante (1995, p. 18). Au terme de son parcours, Cohen conclut de façon un peu circulaire que l'importance qu'il prête à l'égalité (et à la redistribution des revenus qu'elle implique selon lui) ne peut que l'amener à rejeter l'idée de pleine propriété de soi (1995, p. 228). Il est ainsi possible de lire Macrojustice de Kolm (2005) comme se donnant, entre autres, pour objectif d'achever le programme que s'était donné Cohen dans Self-Ownership, Freedom and Equality.

Plutôt que s'en tenir à un refus de principe, Kolm justifie celui-ci en développant une approche qui prétend être le fondement jamais aussi clairement explicité de toutes les positions en faveur de la redistribution des revenus défendues par les égalitaristes libéraux. Pour être un peu plus précis, Kolm va être conduit à montrer que la redistribution des revenus issus du travail individuel ne contrevient pas à la liberté individuelle en élaborant une théorie du « démembrement de la propriété de soi » (idée qui doit être comprise en référence à ce que le droit appelle « démembrement » de la propriété d'un bien).

L'importance du démembrement de la propriété de soi dans la théorie économique de la justice développée par Kolm a été jusqu'à présent largement sous-estimée par les articles portant sur le modèle ELIE (pour " Equal-Labor Income Equalization ») de redistribution des revenus. En dépit du rejet explicite par Kolm de la pertinence du welfarisme ${ }^{3}$ dans le cadre de la macrojustice, les travaux portant sur ce modèle adoptent en effet une démarche héritée de la tradition (welfariste) de la fiscalité optimale pour le présenter et le discuter (Fleurbaey \& Maniquet, 2011 ; Simula \& Trannoy, 2011a, 2011b). Ce parti-pris les conduit à proposer des voies d'amélioration du modèle ELIE qui reviennent à négliger cette prise de distance avec le welfarisme.

2. Richard Overton est le premier à avoir affirmé la pleine propriété de soi dans son pamphlet intitulé $A n$ Arrow Against All Tyrants (1646). Il est toutefois indiscutable que c'est le texte de Locke qui a fait date dans l'histoire de la pensée.

3. Sen définit le welfarisme comme la doctrine qui affirme qu' « en définitive, le caractère souhaitable (goodness) d'un état du monde dépend de l'ensemble des utilités individuelles de cet état, et - plus précisément (more demandingly) - peut être vu comme une fonction croissante de cet ensemble » (Sen, 1979, p. 464). 
Ce cadre d'interprétation du modèle ELIE, teinté de welfarisme, est accepté par toutes les tentatives d'évaluation des conséquences de ce modèle au moyen de simulations. Ooghe \& Schokkaert (2011), qui posent la question de savoir si ELIE est un système de redistribution inutile et coûteux, affirment ainsi la parenté de leur approche avec celle de Simula \& Trannoy (2011a) (Ooghe \& Schokkaert, p. 237, n. 1). Ces deux auteurs présentent le modèle ELIE de Kolm comme un optimum de premier de rang inaccessible (en raison d'une asymétrie d'information) et devant, par conséquent, laisser la place à une situation certes moins efficace, mais réalisable (Ibid., p. 246-247). Lubrano (2011) accepte l'idée, aussi défendue par Simula \& Trannoy (2011a, 2011b), que le modèle EliE contraint tout individu à travailler au moins un certain temps (Lubrano, p. 279). Or, le seul argument pour défendre une telle lecture du modèle ELIE consisterait à accepter l'idée (fondamentalement welfariste) d'un arbitrage entre efficacité sociale et liberté individuelle - idée à laquelle Kolm s'oppose formellement. Pour leur part, de la Croix \& Lubrano (2011) prennent comme objectif évident et ininterrogé la maximisation intertemporelle du capital humain sous contrainte de redistribution des revenus (de la Croix \& Lubrano, 2011, p. 334) - alors même que toute la démarche de Kolm se construit contre l'idée que l'objectif d'une société est la maximisation du bien-être social ${ }^{4}$.

Le welfarisme inhérent à ces « lectures » explique sans doute que tous ces articles aient sous-estimé l'importance du démembrement de la propriété de soi et aient pris, à tort selon nous, la représentation graphique du modèle ELIE pour l'alpha et l'oméga de la réflexion kolmienne sur la macrojustice, alors qu'il n'en est qu'une partie - qui demeure susceptible d'être mal comprise si on ne la replace pas en cohérence avec la théorie de la liberté individuelle qui la sous-tend. Toutefois, et à la décharge des auteurs de ces articles, il faut bien reconnaître que malgré son rôle de fondement du modèle ELIE, le démembrement de la propriété de soi n'est jamais exposé clairement et d'un seul tenant par Kolm.

Dans cet article, nous nous donnons plusieurs objectifs qui reviennent ensemble à mettre en évidence l'intérêt, l'originalité et la portée de la théorie kolmienne du démembrement de la propriété de soi. Nous reconstruirons cette théorie à partir des éléments disséminés dans Macrojustice (2005) et dans ses «articles satellites », nous montrerons comment cette théorie justifie la représentation graphique du modèle ELIE et nous montrerons aussi que cette représentation graphique n'est pas le dernier mot de Kolm concernant la redistribution des revenus. Une telle reconstruction, qui met en évidence la cohérence interne et de la complexité de l'argumentation kolmienne autour de la propriété de soi, est un préalable indispensable à une possible discussion critique. Cette complexité peut en effet aisément conduire à lui adresser des critiques qui manquent, au final, leur cible. Notre objectif n'est donc pas, ici, de défendre le point de vue de Kolm, mais bien de clarifier sa position.

Dans ce but, nous interrogerons tout d'abord la différence entre démembrement classique de la propriété et démembrement de la propriété de soi (1). Nous pourrons ainsi présenter la représentation graphique de la règle ELIE de redistribution des revenus en mettant en évidence le fait qu'elle n'est rien d'autre que la représentation du démembrement de la propriété de soi (2). Cette représentation graphique posant (au moins) un problème majeur en termes de liberté individuelle, nous serons ensuite conduit à questionner la forme de libéralisme défendue par Kolm et notamment à montrer en quoi celui-ci diffère du libéralisme «classique» (3). Nous achèverons notre parcours en insistant sur le fait que le démembrement de la propriété de soi est un argument s'opposant à toutes les formes de libertarisme (4).

4. Sur le sens du non-welfarisme du modèle ELIE, voir Gharbi \& Meinard, 2015. 


\section{Démembrement et propriété de soi}

Le modèle ELIE de redistribution des revenus trouve à la fois son origine et son fondement dans une réflexion sur la propriété et tout particulièrement sur la propriété de soi. En effet, si l'on admet que les individus sont pleinement propriétaires d'eux-mêmes, il est impossible de justifier une forme quelconque de redistribution des revenus issus du seul travail - attendu que les fruits de mon travail m'appartiennent alors indiscutablement et intégralement. Le démembrement de la propriété de soi apparaît donc comme une condition nécessaire de la construction du modèle ELIE.

Nous expliquerons tout d'abord ce que signifie le terme "démembrement» dans l'expression « démembrement de la propriété de soi » (1.1), avant de nous tourner vers une conséquence majeure de cette idée, à savoir le fait qu'elle conduit à considérer que les individus sont partiellement locataires d'eux-mêmes (1.2).

\subsection{Le « démembrement » de la propriété de soi}

Classiquement, on définit le droit de propriété, ou encore droit en pleine propriété d'un bien, comme comportant trois attributs :

- l'usus (ou jus utendi), le droit d'usage du bien ;

- le fructus (ou jus frutendi), le droit d'en percevoir les fruits et les produits ;

- l'abusus (ou jus abutendi), le droit de disposer de sa propriété comme on l'entend : donation, vente, voire même destruction.

Une séparation du droit de propriété en deux parties distinctes est toutefois possible. C'est le cas classique de l'usufruit, dans lequel une ou plusieurs personnes se voient temporairement attribuer l'usus et le fructus d'un bien appartenant à un tiers qui ne garde, pour sa part, que l'abusus. L'abusus dissocié de l'usus et du fructus, autrement dit le droit qui est complémentaire de l'usufruit, s'appelle le « droit de nue-propriété ». On parle alors de " démembrement de la propriété ».

Dans un tel cas, le seul «propriétaire » du bien est le détenteur de l'abusus: les usufruitiers ne sont pas «propriétaires » du bien dont ils ont l'usage et dont ils jouissent des fruits. C'est pour cette raison qu'ils doivent s'en servir en « bon père de famille », c'est-à-dire payer les charges normales d'entretien et restituer le bien au propriétaire à la fin de la période d'usufruit.

Kolm défend l'idée qu'une personne n'est pas pleinement propriétaire de ses capacités productives et porte atteinte à l'unité de la pleine propriété telle qu'elle est définie par la réunion de l'usus, de l'abusus et du fructus (2005, p. 91). C'est pour cette raison qu'il utilise la formule « démembrement de la propriété de soi » (2007a, p. 71). La démarche consiste en effet à poser à la fois que les capacités productives d'une personne sont un bien et que les attributs classiques de la propriété sont, pour lesdites capacités, toujours en droit dissociés.

Il est extrêmement important de préciser que les « capacités productives » dont parle Kolm (et qui forment l'assiette du modèle ElIE de redistribution des revenus) ne sont pas les capacités de gains salariaux maximaux, mais les capacités productives effectivement utilisées pour obtenir un revenu (Kolm, 2007a, p. 79).

« Les gens comprennent que les personnes qui bénéficient d'une capacité à gagner élevée fournissent une certaine aide à ceux qui n'ont pas cette chance, mais seulement si cette capacité produit en effet un gain, pas si cette personne préfère ne pas travailler ou travailler très peu, donc en taxant son loisir comme s'il produisait ce haut revenu. De même, si quelqu'un préfère un travail plus agréable mais moins rémunérateur qu'un 
autre, c'est ce salaire effectif qui importe et non le salaire potentiel qu'il pourrait obtenir autrement » (Kolm, 2009, p. 48, nous mettons en italiques).

Ainsi un actuaire qui déciderait de devenir instituteur paierait en fonction de ses revenus d'instituteur et pas en fonction de ses revenus (possibles) d'actuaire. Si l'on taxait le seul fait d'avoir certaines capacités et non pas celui de les utiliser pour obtenir un revenu, les porteurs de fortes capacités productives se retrouveraient face à l'alternative d'être soit (1) obligés de travailler, soit (2) de devoir trouver un autre moyen d'acquitter ce droit de rente. Et ces deux possibilités serait pour eux une contrainte et donc une violation de leur liberté sociale ${ }^{5}$. Or la liberté sociale, qui a dans Macrojustice une place principielle, exige que « les personnes ne (soient) pas contraintes par d'autres, individuelles ou en groupes ou institutions » (Kolm, 2006, p. 57).

Tout en notant l'analogie entre les deux formes de « démembrement », dans la mesure où il $\mathrm{y} \mathrm{a}$, dans les deux cas, une fragmentation de l'unité supposée de la totalité formée par l'usus, le fructus et l'abusus, il convient de relever certaines différences. Outre le fait que le démembrement de la propriété de soi n'est pas temporaire comme l'est le droit d'usufruit, on doit remarquer que la ligne de séparation entre les trois attributs de la propriété n'est, en l'occurrence, pas la même. En effet, alors que dans le cas du démembrement classique de la propriété l'usus et le fructus vont à une ou plusieurs personnes et l'abusus à une ou plusieurs autres. Dans le cas du démembrement de la propriété de soi, Kolm entend répartir le fructus entre, d'une part, la personne porteuse de certaines capacités productives (à laquelle reviennent aussi l'usus et l'abusus) et, d'autre part, toutes les autres personnes de la société.

\section{Démembrement classique de la propriété}

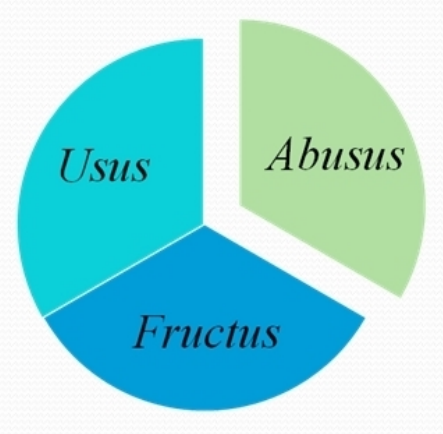

\section{Démembrement de la propriété de soi}

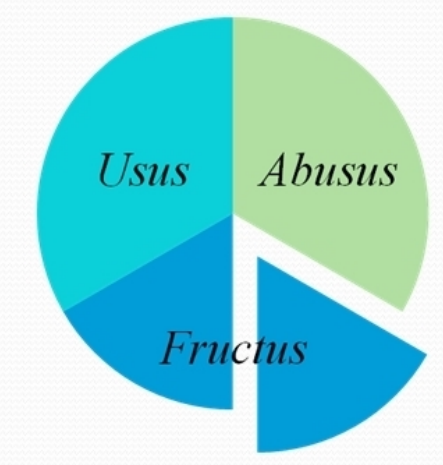

\section{Les deux formes de démembrement de la propriété}

En décomposant la propriété de soi en droits de contrôle (qui comprennent notamment le droit de se vendre ou de se détruire), d'une part, et droit au revenu, d'autre part, Christman (1991) et Taylor (2005) adoptent une position assez proche de celle de Kolm. Ces auteurs ne se réfèrent, toutefois, pas à la tripartition usus/abusus/fructus. De plus, ils défendent l'idée que le droit au revenu d'un individu n'a aucun lien avec son droit de contrôle, alors que Kolm pose qu'une part au moins du fructus de soi est indissolublement liée à son usus, et revient donc en droit à l'individu.

5. Kolm utilise les formules «liberté sociale (social freedom)» et «liberté processuelle (process freedom)» quasiment comme des synonymes. Les deux expressions désignent l'absence de contrainte et renvoient à ce que l'on appelle assez communément « liberté formelle ». Pour cette raison, et afin de faciliter la lecture, nous n'utiliserons que l'expression « liberté sociale » dans la suite de ce texte. 
La question se pose alors de savoir si l'on peut fractionner, comme le fait Kolm, le fructus des capacités productives d'une personne sans fractionner par le fait même son usus et son abusus desdites capacités.

Le fait que l'abusus soit limité ne poserait guère de problème nouveau dans la mesure où l'on se trouverait dans la même situation que lorsque le droit de pleine propriété est partagé en usufruit, d'une part, et en droit de nue-propriété, d'autre part. Dans le cas classique de l'usufruit, le titulaire de la nue-propriété n'a pas le droit de détruire le bien dont il est propriétaire, il voit donc son abusus limité du fait du démembrement de sa propriété, et cela afin de garantir aux usufruitiers la jouissance de leurs droits. Toutefois, il ne semble pas que, dans le cas du démembrement de la propriété de soi, l'abusus de ses propres capacités productives soit limité. Kolm ne mentionne pas, par exemple, d'obligation sociale de la part des individus de ne pas détruire leurs capacités productives en raison du fait qu'une part du fructus de celles-ci revient à la collectivité. Cela est sans doute lié au fait que les capacités productives qui forment la base de la redistribution dans Macrojustice sont les capacités productives effectivement utilisées et pas les capacités productives tout simplement. Détruire ses propres capacités productives consisterait alors à choisir de manière irrévocable de ne plus jamais les utiliser. Le passage du démembrement classique de la propriété au démembrement de la propriété de soi n'implique donc aucune limitation de l'abusus de ses propres capacités productives.

Il est important de préciser que si le démembrement de la propriété de soi n'implique aucune limitation de l'abusus, cela ne signifie pas pour autant que Kolm accepte l'esclavage volontaire - comme le font la très grande majorité des libertariens. Chez Kolm, la liberté individuelle est en effet un principe, et elle ne peut donc pas être abandonnée au profit d'un autre individu : "Le principe n'implique pas [que l'on] ait le droit de se détruire (suicide, drogue, etc.), ni de se vendre ou de se donner (esclavage) » $(1985$, p. 74). Dans les pages suivant cette déclaration, Kolm discute explicitement de la soumission volontaire. Toutefois cette limitation (morale) de l'abusus d'un individu sur lui-même ne découle pas du démembrement de la propriété de soi.

En revanche, l'idée d'un fractionnement de l'usus des capacités productives propres à un individu poserait problème. Le fait que l'usus de soi-même se trouve limité par l'attribution à la personne porteuse d'une capacité productive d'une partie seulement du fructus de celle-ci, amènerait en effet une nouveauté par rapport au cadre de la distinction entre usufruit et droit de nue-propriété : la personne pourrait alors se trouver obligée d'user de ses capacités productives si cela était son seul moyen d'acquitter la taxe y correspondant. L'individu doit donc avoir le plein usus de ses propres capacités productives.

En scindant le fructus des capacités productives d'un individu en deux parties distinctes, le démembrement de la propriété de soi ne limite donc ni l'usus, ni l'abusus de ces capacités. Cela pose toutefois la difficile question de savoir quel rapport un individu entretient avec la part de fructus qui ne lui revient pas.

\subsection{Locataire partiel de soi}

Les capacités productives sont indissociables des personnes qui en sont porteuses - elles ont, en effet, "la propriété particulière d'être a priori incorporées dans des personnes » (Kolm, 2007a, p. 71). On doit donc admettre que n'étant pas pleinement propriétaire de ses capacités productives, une personne n'est pas pleinement propriétaire d'elle-même. Kolm dira qu'elle est partiellement « locataire d'elle-même » (2005, p. 60).

Le locataire d'un bien classique se trouve dans l'obligation d'acquitter un loyer pour obtenir le droit d'user du bien en question et de bénéficier des fruits éventuels de cet usage. Il 
se trouve ainsi face à une alternative : payer un loyer ou renoncer à l'usage du bien dont il n'est pas propriétaire. Nous retrouvons donc, dans le cas de la location d'un bien, le cadre classique de la propriété à ceci près que le fructus est scindé d'une part en "rente » (le loyer) et d'autre part en " bénéfice» (les fruits éventuels de l'usage du bien) ${ }^{6}$. Le loyer qui revient au propriétaire servant, en l'occurrence, à obtenir de ce dernier qu'il cède pour une période déterminée l'usus et le fructus restant du bien loué.

Dès lors que l'on ne parle plus d'un objet mais des capacités productives d'un individu, la situation est différente. Nous avons vu qu'il est tout à fait impossible d'obtenir l'usus des capacités productives dont une autre personne est porteuse (Kolm, 2005, p. 92). Cela reviendrait, en effet, à avoir le droit de décider des actions de cette personne à sa place, ce qui violerait sa liberté sociale - c'est-à-dire sa liberté d'agir et de choisir le but de son action. C'est cette raison qui conduit à affirmer que toute personne est « locataire en titre d'ellemême » (Kolm, 2007a, p. 72), autrement dit qu'elle est la seule à pouvoir user de ses capacités productives et cela même si elle prend le parti de ne pas le faire.

Toutefois, il faut bien comprendre que cette exclusivité quant à ses propres capacités productives ne signifie pas qu'un individu ne puisse rien céder de l'usage qu'il fait de cellesci - car alors le fait du salariat serait incompréhensible.

La nécessité pour une théorie de la liberté de penser le salariat se trouvait déjà dans Kolm (1985). Dans sa discussion des thèses de Rawls, Kolm affirmait :

« il y a certaines libertés que l'on abandonne sans cesse. Par exemple lorsqu'on se loue pour un salaire. Rawls ne va certes pas jusqu'à condamner cela, mais il ne précise guère où s'arrêtent ces libertés de base. C'est une interprétation intéressante de la théorie de l'exploitation de Marx que de dire que celui-ci la trouve immorale parce qu'il adhère à une éthique rawlsienne avec une conception extensive de la liberté - puisque le salariat est alors condamnable » (1985, p. 382).

L'argument met clairement Rawls en demeure de choisir entre une conception absolue de la liberté, qui le conduirait, avec Marx, à condamner le salariat, ou une conception relative, qui correspondrait point pour point à celle de Kolm.

Le salariat n'est pas à proprement parler l'acquisition par l'employeur de l'usus des capacités productives du salarié, sinon ce dernier abdiquerait sa liberté et serait ainsi comme dépossédé de lui-même durant la période où il est employé. A la différence d'un outil, qui n'a aucune volonté propre et, par conséquent, aucune liberté, un salarié choisit librement d'accomplir chacune de ses actions, il n'est pas contraint d'agir. Il reste donc titulaire du plein usus des capacités productives qu'il met à la disposition de son employeur. Ce que le salarié cède contre le salaire qu'on lui verse, c'est seulement l'usage qu'il fait lui-même de ses capacités et une partie des fruits qui en découlent pour une période définie.

Il faut donc distinguer, d'une part, un usus « originel $»^{7}$ qui appartient en propre au porteur des capacités productives, et un usus «secondaire » qui pourrait être cédé. Et on doit ajouter que le second dépend du premier, mais non l'inverse, que l'usus « primaire » est condition nécessaire, mais pas suffisante, de l'usus «secondaire ». Pour le dire autrement, et en se référant à la distinction entre liberté «formelle» et liberté « réelle», on pourrait qualifier l'usus primaire de « formel », dans la mesure où il est un droit absolu, mais qui reste

6. Il n'y a pas lieu d'opposer cette distinction bipartite entre droit de rente et droit aux bénéfices à la tripartition usus/abusus/fructus, vu que la première s'insère dans la seconde : « Le droit aux fruits (jus frutendi) englobe les droits aux bénéfices et les droits de rente » (Kolm, 2005, p. 91).

7. «La liberté sociale implique d'avoir le droit d'usage originel de ses capacités personnelles » (Kolm, 2006b, p. 490, les italiques se trouvent dans le texte de Kolm). 
de pure forme sans l'usus secondaire. L'usus secondaire pourrait alors être qualifié d'usus « réel ».

« La liberté sociale des personnes implique que celles-ci aient a priori le droit d'usage en leurs propres capacités. En effet, décider de l'usage de capacités de quelqu'un est commander son action. Cela étant, une personne peut louer un droit d'usage sur ses capacités, le vendre pour un temps. C'est le salariat. Mais elle doit en détenir le droit primaire, de base, initial, si elle est libre. » (Kolm, 2007a, p. 71).

Alors que la personne « doit » détenir l'usus primaire d'elle-même, c'est-à-dire ne peut pas ne pas le détenir (si elle est libre), elle «peut » en céder l'usus secondaire. Elle peut donc être salariée sans perdre sa liberté - puisqu'elle est alors la seule à faire (et à pouvoir faire) usage d'elle-même.

La distinction entre usus primaire et usus secondaire permet de comprendre qu'une personne puisse mettre l'usage qu'elle fait elle-même de ses capacités productives au service d'une autre sans abdiquer, par le fait même, sa liberté. Elle permet aussi de comprendre qu'une personne puisse se trouver locataire d'elle-même. En effet, si louer un bien revient à payer un loyer contre le droit d'usage et la part de fructus qui découle de cet usage, être locataire de soi, fût-ce partiellement, ne peut pas signifier qu'on loue le droit d'usage primaire de ses propres capacités productives - cela reviendrait en effet à nier notre liberté. Si donc le porteur de capacités productives doit acquitter un loyer, ce ne peut pas être pour obtenir, comme dans le cas de la location classique, le plein usus et la part de fructus qui correspond aux bénéfices - car l'usus primaire lui est acquis par principe.

Mais, dans ce cas, que loue le locataire de lui-même ? Il ne pourrait pas être satisfaisant de dire qu'il loue seulement la part de fructus qui correspond aux bénéfices, dans la mesure où le bénéfice est indissolublement lié à l'usus. Ce qu'il loue, c'est justement l'usus secondaire. Le fait d'être locataire en titre de soi-même assure à l'individu de toujours pouvoir (et, encore une fois, d'être le seul à pouvoir) utiliser ses capacités productives, mais elle n'exclut pas que ladite personne ait à verser une contrepartie si elle désire les utiliser.

Autrement dit, un individu, en tant que locataire en titre de lui-même, a le choix entre utiliser ou pas ses capacités productives - sur ce plan, il est totalement libre. Et puisqu'il a le choix de les utiliser ou pas, il possède bien, formellement, le plein usus de celles-ci (dans la mesure où la distinction entre usus primaire et secondaire n'est pas une partition ou une séparation en deux parties quantitativement distinctes, mais une distinction de raison). Ceci étant dit, s'il choisit d'utiliser ces capacités, dont il n'est pas pleinement propriétaire (il ne possède pas le plein fructus de lui-même), il se trouve dans l'obligation d'acquitter un loyer. Ce n'est donc qu'une fois qu'il a décidé d'utiliser ses capacités qu'il doit acquitter une taxe, un droit de rente sur leur valeur. Il reste donc totalement libre d'utiliser ses capacités productives pour transformer son travail en revenu ou pas, et garde ainsi la possibilité de ne pas « louer » ce bien s'il choisit de ne pas en jouir.

« La liberté [sociale] équivaut à avoir le droit d'usage et le droit aux bénéfices [qui correspondent aux fruits éventuels de cet usage] de ses propres capacités » (Kolm, 2005, p. 61) - car le contraire reviendrait à ne pas être libre d'agir. Mais elle n'implique pas la pleine propriété de soi (Kolm, 2006, p. 58). Ainsi, un individu peut bien payer une rente pour obtenir l'usage réel de ses capacités et les fruits de cet usage sans que cela remette en cause sa liberté sociale puisque l'usus pour lequel il paie n'est pas l'usus " primaire » qui s'identifie avec ladite liberté, mais l'usus « secondaire » qui dépend du premier sans s'y identifier et qui peut donc, sans difficulté, faire l'objet d'une transaction - comme c'est le cas dans le salariat, notamment. Dans cette analyse, le salaire de l'individu correspond donc à la part de fructus appelée «bénéfices » (partie du fructus revenant, comme l'usus et l'abusus, à l'individu dans 
notre schéma, page 5), c'est-à-dire à la totalité du fructus à laquelle on retire le " droit de rente " (partie du fructus ne revenant pas à l'individu, dans le même schéma). Il n'y a donc aucune contradiction à acquitter un droit de rente équivalent à la valeur d'une partie de mes capacités productives pour obtenir l'usus «secondaire » de ces capacités dont je suis porteur, mais pas pleinement propriétaire.

La question se pose toutefois de savoir si la distinction que nous venons d'établir entre usus primaire et usus secondaire ne remet pas en cause l'idée que je possède le plein usus de moi-même, car sans cela je ne serais pas libre d'agir comme je le souhaite.

Ce n'est pas le cas. En effet le propos n'est pas ici de scinder l'usus des capacités productives en deux parties quantitativement distinctes qui ne reviendraient pas en droit à leur porteur. Contrairement à la distinction en deux parties du fructus dans le démembrement de la propriété de soi (ou à la fragmentation de l'usus dont nous avons discuté dans la section 1.1), la distinction entre usus primaire et usus secondaire n'est pas une séparation en deux parties, mais une distinction de raison : l'usus primaire est la totalité de l'usus comprise formellement et l'usus secondaire est la totalité de l'usus comprise réellement. Le droit de rente que le porteur de capacités productives doit acquitter correspond ainsi à une partie de la valeur de ses capacités et est une partie du fructus de celles-ci. Le plein usus revient donc bien en droit au seul porteur. Il ne doit s'acquitter d'une taxe, c'est-à-dire abandonner une partie du fructus de ses capacités, que s'il les utilise réellement.

La distinction des usus primaire et secondaire permet à la fois de poser que les individus sont libres et qu'ils doivent acquitter un «droit de rente» (Kolm, 2005, p. 61) pour pouvoir utiliser leurs capacités productives :

« Si [un agent] possède un droit de rente sur la capacité [d'un autre], le porteur doit acquitter la rente correspondante [pour utiliser sa capacité]. Cette somme peut être vue comme le prix payé pour la mise à disposition des services que cette capacité peut fournir. Cette mise à disposition permet au porteur d'user librement de cette capacité et de récolter les bénéfices qui en découlent, quelle que soit leur nature. Le porteur achète ainsi un droit d'user de sa capacité et de bénéficier de cet usage. A raison de ce droit de rente [...], il est seulement locataire de lui-même » (Kolm, 2005, p. 92).

Cette distinction nous met face au paradoxe que les individus doivent acquitter une forme de loyer pour user de capacités dont ils sont, pourtant, les seuls propriétaires. En effet, les titulaires de la partie du fructus de mes capacités productives qui ne me revient pas en droit ne sont pas «propriétaires » de celles-ci, pas même partiellement : ils en sont tout au plus les «fruitiers partiels $»^{8}$. Ce paradoxe ne confine pas à la contradiction : il ne serait pas absurde que l'un des usufruitiers d'un bien paie les autres pour obtenir concrètement et totalement l'usus et le fructus de ce bien. Mutatis mutandis, le porteur de capacités productives, en tant que fruitier partiel, se trouve dans l'obligation de verser une rente s'il veut obtenir l'usus réel de ses capacités productives et, par conséquent, le fructus-bénéfice qui en est corrélatif (mais il n'y est absolument pas obligé s'il ne le souhaite pas).

La redistribution des revenus issus du travail n'implique donc pas, comme le prétend Michael Gorr de façon provocante, que nous soyons seulement " actionnaires majoritaires » de nous-mêmes (1995, p. 271) et que la société soit, par conséquent actionnaire minoritaire de nous - voir aussi Nozick (1974, p. 172). En effet, les actionnaires d'une entreprise en sont partiellement propriétaires, ce qui n'est pas le cas de la société dans le démembrement de la propriété de soi défendu par Kolm (qui ne donne aux autres membres de la société que le

8. Cette formule nous a été suggérée par Alain Wolfelsperger. 
statut de « fruitiers partiels » des capacités productives d'un individu et donc n'en fait pas des « propriétaires », fût-ce partiellement).

L'alternative que nous avions rencontrée dans le cas de la location classique d'un bien entre payer le loyer ou renoncer à l'usage de celui-ci est reconduite dans la location de soi : l'individu doit choisir entre acquitter une taxe sur ses capacités productives ou renoncer à les utiliser.

Avec le démembrement de la propriété de soi, Kolm pense thématiser un aspect qui demeurait jusque-là presque totalement implicite dans les théories dites «égalitaristes libérales »-caché sous la thèse de la propriété collective des capacités individuelles ou de la solidarité dans une société. Rawls défend bien l'idée d'une mise en commun des capacités productives individuelles : «Le principe de différence représente, en réalité, un accord pour considérer la répartition des talents naturels comme un bien commun (common asset) et pour en partager les bénéfices quelle que soit leur répartition [initiale] » (Rawls, 1971, p. 132, nous modifions la traduction et ajoutons la précision entre crochets). Ou encore « Les deux principes [de justice] reviennent [...] à prendre l'engagement de considérer la répartition des aptitudes individuelles comme un bien collectif (collective asset) » (Rawls, 1971, p. 209, nous modifions la traduction $)^{9}$. Toutefois, et bien que sa position semble faire signe vers un tel démembrement de la propriété de soi, Rawls ne traite jamais directement des implications et des difficultés de cet aspect en termes de liberté individuelle. De même, les développements de Christman (1991) et Taylor (2005) semblent appeler un tel démembrement sans jamais aborder les difficultés qui lui sont inhérentes.

Le démembrement de la propriété de soi constitue l'analyse des éléments que présuppose nécessairement la thèse de la propriété collective des capacités productives. L'enjeu de ce travail d'analyse associé au démembrement de la propriété de soi (corrélat nécessaire d'un système d'imposition basé sur les capacités productives individuelles) est de clarifier le sens et les implications de cette position classique chez les égalitaristes libéraux et ainsi de fournir une nouvelle assisse à la justification libérale de la redistribution des revenus issus du travail.

\section{Le modèle ELIE de redistribution des revenus}

L'idée centrale de Macrojustice est que la justice sociale requiert d'égaliser les libertés des individus. En focalisant son attention sur la liberté, Kolm prend ses distances avec les approches welfaristes ${ }^{10}$ qui concentrent toute leur attention sur le bien-être (welfare), et qui identifient ainsi la justice sociale à un problème de maximisation des fonctions d'utilité individuelles et collective. Cette prise de distance l'amène à défendre l'idée d'une imposition qui ne se baserait pas sur le revenu total effectif des individus, mais qui serait forfaitaire et porterait sur la valeur économique des capacités productives effectivement mobilisées par les individus dans le cadre de leur travail.

Pour comprendre ce que Kolm entend par la formule «égaliser les libertés des individus ", il est nécessaire de distinguer au préalable la liberté sociale de la liberté de choix. Nous l'avons dit, la liberté sociale se définit par le fait que «les personnes ne sont pas contraintes par d'autres, individuelles ou en groupe ou institutions », ce qui implique «que les personnes doivent être contraintes de ne pas contraindre les autres si elles ne s'en abstiennent pas volontairement »(2006, p. 57). Autrement dit, la liberté sociale se caractérise

9. A ce sujet voir aussi Kernohan (1990).

10. « Le critère pertinent [de la redistribution des revenus] au niveau global se révèlera être non le bien-être mais la liberté » (2007a, p. 62). 
(qualitativement) par la nature des contraintes qui pèsent sur les individus: les libertés sociales des individus sont, par définition, égales et ne peuvent donc pas se trouver en concurrence. Les libertés de choix des différents individus se définissent, quant à elles (quantitativement) par l'ampleur des choix qu'elles rendent possibles et peuvent donc se trouver en conflit. La liberté que Kolm entend égaliser est ainsi la liberté de choix.

La distinction qu'opère Kolm entre liberté sociale et liberté de choix correspond à celle, classique, de la liberté formelle et de la liberté réelle. Et cela signifie que, selon lui, la première est une condition nécessaire, mais pas suffisante de la seconde $(2006$, p. 67 ; 2007a, p. 69). La liberté réelle se définissant comme la liberté formelle à laquelle on ajoute les moyens réels d'agir, la liberté de choix est la liberté sociale à laquelle on ajoute les moyens de choisir réellement, autrement dit un revenu ${ }^{11}$. La façon dont Kolm pose ce problème exclut que les libertés de choix en question puisse être égalisées par le biais d'une imposition des revenus effectivement gagnés ou, pour le dire autrement, des résultats du travail (car il s'agirait d'une violation de la liberté sociale des individus ainsi imposés). L'assiette de redistribution ne pourra donc être basée que sur les moyens d'obtenir un revenu, c'est-à-dire sur les capacités que les individus mobilisent pour obtenir un revenu. Si les individus étaient entièrement responsables de leurs capacités productives, l'idée de procéder à une telle égalisation de leurs libertés de choix serait difficile à justifier - mais Kolm ne le considère pas.

L'argument mobilisé ici évoque celui de Rawls lorsqu'il faisait remarquer que personne ne mérite son mérite (Rawls, 1971, p. 349-350). La critique par Rawls de la méritocratie est liée à son idée de propriété commune des capacités productives individuelles, dans la mesure où l'arbitraire du mérite est un argument extrêmement puissant en faveur de la socialisation d'une partie, au moins, des talents. De même, Kolm pose que si l'on a, certes, la responsabilité de l'acquisition d'une partie de nos capacités productives, une autre part, non négligeable, ne dépend pas de nous, mais nous est à proprement parler « donnée ». C'est pour cette raison qu'il considère une partie des capacités productives individuelles (celles dont nous ne sommes pas responsables) comme des ressources données à la société au même titre que les ressources naturelles. Et dès lors l'idée d'une égalisation de celles-ci apparaît moins difficile à admettre.

Le principe de redistribution des revenus préconisé par Kolm consiste à opérer un « partage égal du produit du même travail » (Kolm, 2007a, p. 64) ou encore une « égalisation des revenus issus d'un travail égal ${ }^{12} »$ (ELIE, pour Equal-Labour Income Equalization). Il va de soi que cette formule mérite une explication. Le travail égal dont il est question est un travail « égal » dans le sens où il s'agit de la même durée d'un travail différent. Le travail fourni par les différents individus au cours d'un temps de travail identique serait ainsi rémunéré de façon identique après égalisation - en dépit de toutes les différences qui peuvent exister entre les différentes fonctions qu'occupent les différents individus. Ainsi, un conducteur d'autobus et un chirurgien verraient leurs revenus salariaux égalisés pour une période donnée qui serait fonction d'un paramètre de redistribution $k$, compris entre 0 et 1 inclus, et leurs revenus ne diffèreraient que pour leur travail effectué au-delà de cette période.

11. Il est essentiel de noter que cette liberté, qui est déterminée par le revenu, est seulement une liberté économique - pas une liberté politique, et encore moins une quelconque liberté métaphysique. On pourrait même être tenté de la qualifier de "condition de la liberté" plutôt que de "liberté" à proprement parler. L'égalisation des libertés que vise à garantir la construction de Kolm est ainsi nettement moins ambitieuse que celle se trouvant au centre de l'approche par les capabilités de Sen (1980): elle est strictement économique et constitue une condition nécessaire d'une liberté comprise de manière plus extensive.

12. Nous reprenons la traduction proposée par Gamel (2005, p. 184). 
Ce nouveau schème de redistribution des revenus proposé par Kolm soumettrait ainsi chacun des membres de la société à un transfert de revenu (positif ou négatif selon les cas) proportionnel à la différence entre ses propres capacités productives et les capacités productives moyennes. Si l'on note :

- $\quad k$, le coefficient de redistribution des revenus d'une structure ELIE ;

- $w_{i}$, les capacités productives ou le taux de salaire ${ }^{13}$ de l'individu $i$;

$-\bar{w}$, les capacités productives moyennes telles que $\bar{w}=(1 / n) \Sigma w_{i}$ (avec $n$ le nombre d'individus) ;

- $\quad l_{i}$, le temps de travail librement choisi par l'individu $i$ (normalisé de 0 à 1$)$;

$-\lambda_{i}$, le temps de loisir de l'individu $i$ (on a donc $\lambda_{i}=1-l_{i}$ );

alors un individu $i$ gagnerait, avant redistribution, $w_{i} l_{i}$. Selon le modèle ELIE, il transfèrerait ensuite à la société la somme équivalant à $k w_{i}$ et recevrait d'elle $k \bar{w}$ comme salaire égalisé pour le temps de travail $k$.

Il va de soi que représenter les capacités productives d'un individu par son seul salaire horaire (ou taux de salaire), et donc par une droite, est une approximation - la productivité marginale d'un individu devant probablement être considérée comme décroissante. Si l'on excepte les salaires des "grands patrons", qui relèvent selon Kolm d'un effet de club et par conséquent n'ont pas grand chose à voir avec le marché du travail, le taux de salaire est une variable empiriquement mesurable et qui résume un grand nombre de dimensions du travail (formation nécessaire, expérience mobilisée, intensité du travail lui-même, etc.) (Kolm, 2005, chap. 10). Pour cette raison, Kolm argue que cette approximation « fournit une structure claire, simple et suffisante pour discuter la logique, les raisons morales, les conditions, le sens et les propriétés des distributions ELIE » (2005, p. 145). Kolm présente toutefois un schéma plus raffiné de la structure ELIE dans lequel les courbes de productivités des individus sont des fonctions strictement concaves (2005, p. 210).

Le graphique de la page suivante (adapté de Kolm, 2005, p. 157) représente une société extrêmement simple ne comportant que deux individus dont les capacités productives diffèrent. Il montre que sans transfert le revenu de la personne moins productive que la moyenne (identifié par l'indice $p p$ pour signifier " peu productive ») est égal à $w_{p p} l_{i}$, de telle sorte que son revenu avant redistribution est compris entre 0 et $w_{p p}$. Après redistribution, cette personne moins productive que la moyenne bénéficie d'un transfert $t_{p p}$ égal à $k\left(\bar{w}-w_{p p}\right)$, positif (puisque, par hypothèse, $\bar{w}>w_{p p}$ ), et son revenu maximal passe de $w_{p p}$ à $y_{p p}\left(\right.$ avec $\left.y_{p p}=k \bar{w}+\left(l_{i}-k\right) w_{p p}\right)$. Cette translation augmente le domaine de choix, et donc la liberté de choix, de cette personne. Il lui est possible d'atteindre un revenu supérieur à celui qu'elle pouvait atteindre dans le meilleur des cas avant la redistribution $\left(w_{p p}<y_{p p}\right)$. De plus, quelle que soit la période de temps consacrée à travailler, le revenu de cette personne moins productive que la moyenne augmente $\left(k \bar{w}+\left(l_{i}-k\right) w_{p p}>w_{p p} l_{i}\right)$.

Sans transfert, le revenu de la personne plus productive que la moyenne (identifié par l'indice tp pour signifier « très productives ») est, pour sa part, égal à $w_{t p} l_{i}$, de telle sorte que le revenu de cette personne est avant transfert redistributif compris entre 0 et $w_{t p}$. Après redistribution, cette personne très productive « bénéficie », si l'on peut dire, d'un transfert $t_{t p}$ égal à $k\left(\bar{w}-w_{t p}\right)$, négatif (puisque, par hypothèse, $\bar{w}<w_{t p}$ ), et son revenu maximal passe de $w_{t p}$ à $y_{t p}\left(\right.$ avec $\left.y_{t p}=k \bar{w}+\left(l_{i}-k\right) w_{t p}\right)$. Quel que soit son temps de travail $l_{i}$, pour la personne

13. Le fait de prendre le taux de salaire d'une personne comme base du calcul n'est pas un abandon de l'idée selon laquelle les capacités productives constitueraient une meilleure assiette fiscale que le revenu effectivement gagné. En effet, le taux de salaire est uniquement fonction des capacités productives utilisées par l'individu pour obtenir un revenu, ce que son revenu total effectif n'est pas forcément : il dépend aussi du temps de travail. 
plus productive que la moyenne ce transfert négatif correspond à une perte de revenu $\left(k \bar{w}+\left(l_{i}-k\right) w_{t p}<w_{t p} l_{i}\right)$ et donc à une diminution de son domaine de choix.

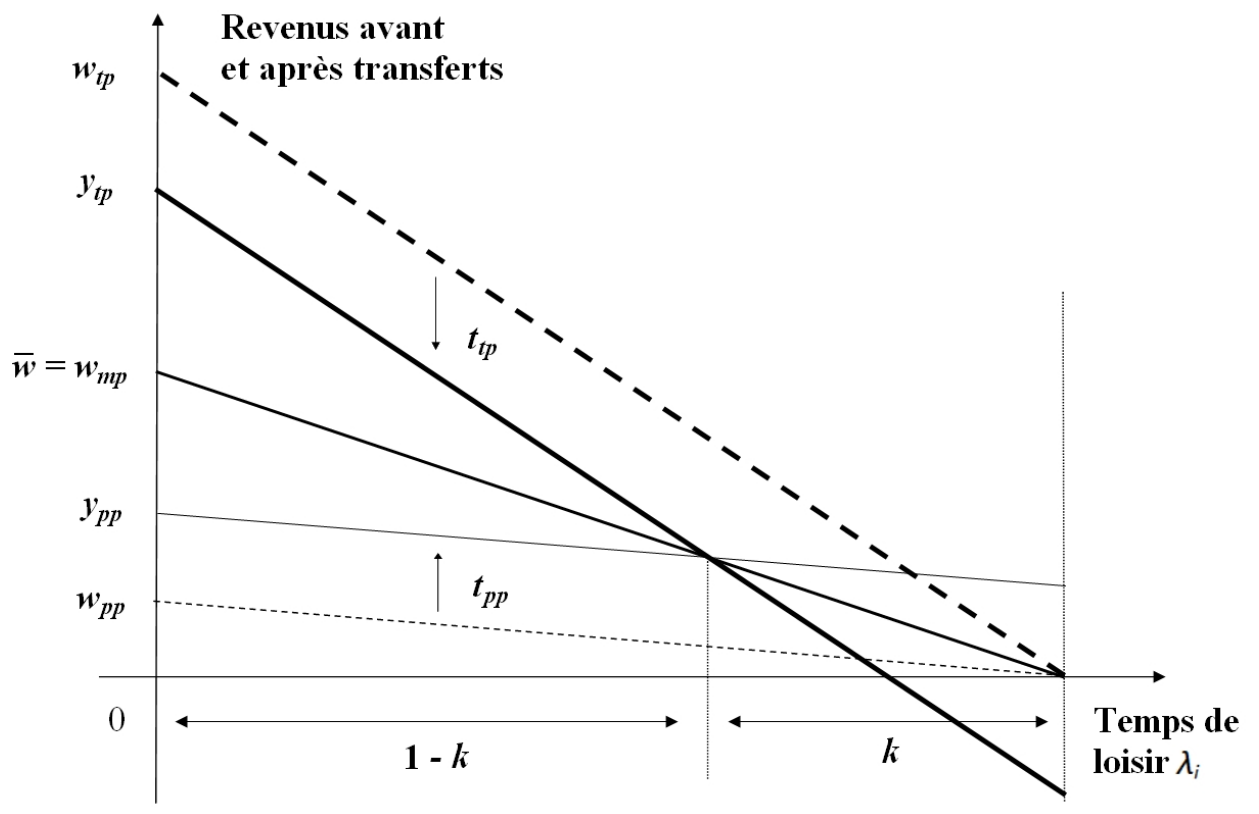

\section{La redistribution des revenus d'après le principe ELIE}

Enfin, la droite qui relie un revenu nul pour un temps de travail nul à un revenu $\bar{w}$ pour un temps de travail égal à 1 correspond à la droite de budget d'un individu (en l'occurrence hypothétique puisque notre société ne comprend que deux individus) qui aurait des capacités productives exactement égales aux capacités productives moyennes. Un tel individu hypothétique ne bénéficierait de (ou ne serait soumis à) aucun transfert.

Le résultat de la translation des droites de budget des individus en fonction de la différence entre leurs capacités productives et les capacités productives moyennes est que toutes les droites de budget dans l'espace revenu-loisir se coupent en un même point qui correspond à $\lambda_{i}=1-k$. L'unicité de ce point de rencontre des droites de budget individuelles après redistribution ne dépend absolument pas du fait que notre graphique représente une société composée de seulement deux individus : le transfert dépendant uniquement de la différence entre les capacités productives d'un individu et les capacités productives moyennes, ce point de rencontre est unique quel que soit le nombre d'individu. Les ressources dont peuvent disposer les individus déterminent leur domaine de choix possible, qui détermine à son tour leur liberté de choix. Dans cette mesure, le modèle EliE égalise donc bien les libertés de choix des individus (puisqu'un individu qui n'aurait aucun revenu n'aurait, en termes économiques, aucune liberté réelle d'action) : au point $\lambda_{i}=1-k$, tous les individus ont la même liberté de choix - même si en deçà et au-delà de ce point leurs libertés diffèrent.

En fait d' « égalisation des revenus issus d'un travail égal », la structure de redistribution ELIE est ainsi une égalisation des revenus issus de capacités productives différentes pour une durée de travail $k$ identique. Pour insister encore un peu plus, dans le modèle ElIE, les capacités productives deviennent les seules variables soumises au prélèvement fiscal, indépendamment du temps de travail : la variable $l_{i}$ désignant le temps de travail librement choisi par l'individu $i$ ne se trouve pas dans la formule qui détermine le montant du transfert auquel il est soumis et qui est de la forme $t_{i}=k\left(\bar{w}-w_{i}\right)$. 
Selon Kolm, l'intérêt de ce déplacement d'une imposition des gains totaux effectifs vers une imposition des capacités effectivement mobilisées pour obtenir un revenu est de rendre les assiettes fiscales inélastiques: elles ne varieraient pas en fonction des comportements individuels. Une telle inélasticité de l'assiette fiscale garantirait alors que le prélèvement n'entraîne pas de distorsion dans le comportement des agents et donc d'inefficacité au sens de Pareto.

Mais si l'on revient à notre exemple d'un actuaire qui déciderait de devenir instituteur, ne peut-on pas penser que le système d'imposition pèse dans sa décision et ainsi modifie son comportement? A cette question, Kolm répond que ce que l'on entend habituellement par " élasticité de l'assiette fiscale» est le fait de réduire son temps de travail en raison du système d'imposition. De ce point de vue, le modèle ELIE est effectivement inélastique, dans la mesure où la modification du temps de travail d'un individu ne modifierait en rien son imposition. Quant à une personne qui choisirait de changer de travail, cela ne peut pas être pris en compte par un système d'imposition, car cela irait contre la liberté sociale des individus. Pour le dire d'un mot, dans la mesure où Kolm ne considère pas la maximisation de l'utilité sociale comme l'objectif d'une société, cette (possible) perte en termes d'utilité sociale serait alors le prix de la liberté individuelle - qui, en tant que principe, ne peut être soumise à aucun arbitrage et à aucune contrainte.

Il est essentiel de noter que le modèle ELIE n'est rien d'autre que la représentation graphique du démembrement de la propriété de soi. En effet, le démembrement de la propriété de soi distingue dans les revenus issus du travail d'un individu (1) une part "socialisée ", jugée ne pas dépendre du tout de la responsabilité individuelle, vouée à prélèvement intégral et à redistribution égalitaire entre les membres de la société, et (2) une part jugée à l'inverse dépendre intégralement de la responsabilité individuelle, et pour laquelle le principe de la propriété de soi s'applique donc. C'est d'ailleurs pour cette raison que Kolm appelle parfois $(1-k)$ le « degré de propriété de soi » $(2005$, p. $163 ; 2006$, p. 73$)$.

Un aspect de ce graphique mérite toutefois qu'on lui accorde une attention particulière : la translation vers le haut de la droite de revenus de la personne moins productive que la moyenne semble impliquer que celle-ci obtiendrait un revenu positif en ne travaillant pas. En fait, dans la mesure où la structure ELIE de redistribution des revenus est une « réciprocité générale équilibrée du travail » (Kolm, 2007a, p. 76), une personne qui choisirait de ne pas travailler ne pourrait pas bénéficier d'un transfert positif.

Le cas du chômeur involontaire serait différent : il serait considéré comme travaillant à temps plein mais ayant, temporairement, des capacités productives $w_{i}$ égales à $0-\mathrm{il}$ bénéficierait donc pour sa part de l'égalisation des revenus :

« La méthode technique consiste à assimiler une impossibilité à trouver plus d'emploi à une impossibilité à gagner plus avec plus de travail. [...] Le résultat est qu'un chômeur involontaire reçoit [...] un revenu $k \bar{w}$ si son chômage involontaire est total ou si c'est un chômeur involontaire partiel qui ne peut pas travailler plus que $k »$ (Kolm, 2006, p. 76).

Dans la pratique, cela pose toutefois le difficile problème de savoir comment déterminer si un chômeur choisit sa situation ou pas.

Même si l'on omettait cette difficulté (qui est loin d'être négligeable), il resterait encore que la personne plus productive que la moyenne se trouverait dans l'obligation de travailler pour parvenir à un revenu nul. Et une telle situation correspondrait à une sorte d' « esclavage des talentueux » qui semble difficilement compatible avant l'insistance de Kolm sur le fait que la liberté individuelle est le principe de toute sa réflexion. 


\section{Un libéralisme peu « classique »}

Le modèle ELIE, qui découle de la théorie kolmienne du démembrement de la propriété de soi est-il conduit à défendre une forme d'esclavage des talentueux ? Une telle question nous oblige à revenir vers les aspects philosophiques de la démarche de Kolm et à interroger plus précisément le sens qu'il donne à son « libéralisme »- le terme « libéralisme » étant ici entendu comme "position qui fait de la liberté individuelle un principe échappant à toute forme d'arbitrage $»$.

Dans un premier temps, nous nous demanderons si le modèle ELIE correspond à une forme d'esclavage des talentueux (3.1), ce sera notamment l'occasion de mettre en évidence le fait que le graphique du modèle ELIE que nous venons de présenter n'est pas le terme dernier de la réflexion de Kolm quant à la redistribution globale des revenus dans une société. Après avoir expliqué comment Kolm entend écarter cette objection, extrêmement gênante dans une perspective qui se prétendrait «libérale », nous reviendrons sur l'articulation des concepts de liberté sociale et de propriété de soi et sur les caractéristiques du libéralisme qu'adopte Kolm (3.2).

\subsection{Le problème de l'« esclavage des talentueux »}

Lorsque les individus talentueux sont contraints de travailler énormément du fait que leur loisir a un très fort coût d'opportunité, c'est-à-dire du fait de la valeur marchande de leur temps lorsqu'il est utilisé de façon économiquement productive, on parle, selon la formule rendue célèbre par Dworkin, d' " esclavage des talentueux » (1981, p. 312). Dans un tel cadre, les personnes les plus productives sont obligées de l'être autant que possible. L'utilisation du terme « esclavage " se justifie parce qu'elles n'ont pas, alors, la possibilité de choisir de travailler moins et que leurs efforts ne leur profitent pas.

La première remarque concernant l' " esclavage des talentueux » dans Macrojustice est que l'on pourrait difficilement accuser Kolm ne pas avoir pensé à ce cas puisqu'il utilise cette formule dès l'introduction de l'ouvrage $(2005$, p. 28$)$ et qu'il la mentionne par la suite à de nombreuses reprises ${ }^{14}$. Il donne de cette situation qui soumet les individus les plus talentueux à une injuste exploitation sociale une définition précise : elle correspond selon lui à une redistribution intégrale des revenus.

Il y a deux raisons, dont chacune est suffisante seule, qui conduisent à rejeter l'idée d'une telle redistribution intégrale des revenus. La première, que l'on pourrait qualifier de « philosophique », est qu'une telle redistribution violerait la liberté sociale des individus les plus talentueux. Si l'on définit, en effet, la liberté sociale comme absence d'interférence contraignante, le fait de se trouver dans l'obligation de travailler en constitue une violation : «Forcer les gens à travailler violerait la liberté sociale » (Kolm, 2006, p. 59). La seconde raison est, quant à elle, strictement économique : un tel système serait Pareto-inefficace. Toutes les personnes gagneraient, en travaillant à temps plein, exactement le même salaire quelles que soient leurs capacités productives respectives. Dès lors, les individus les plus productifs seraient fortement incités à masquer leurs capacités productives et à les minorer cela reviendrait pour eux à une diminution de leurs efforts de travail sans diminution de leurs revenus. Bref, pour le dire d'un mot, un système de redistribution qui concernerait la totalité des revenus « couperait toute incitation matérielle à travailler» (Kolm, 2006, p. 69).

La question se pose à présent de savoir si l'on ne pourrait pas, contre l'avis de Kolm, considérer qu'une redistribution forfaitaire (basée sur les capacités productives individuelles)

14. Voir notamment Kolm, 2005, p. 132, p. 211, p. 246, p. 254, p. 295, p. 323, p. 334, p. 443. 
et ne concernant pas la totalité des revenus, tombe elle aussi dans la catégorie de l'« esclavage des talentueux ». Une telle redistribution des revenus, du fait de son aspect égalisateur (forfaitaire) réduirait forcément le domaine de choix des individus les plus productifs pour pouvoir augmenter celui des moins productifs. Ce serait donc bien ces individus plus productifs qui porteraient le poids de la redistribution.

Le premier point que l'on doit avancer contre cette idée selon laquelle la structure générale de redistribution des revenus ELIE se ramènerait en dernière instance à un « esclavage des talentueux» concerne la signification générale que l'on donne à la redistribution. Le but du modèle ELIE est notamment de garantir un revenu minimal $k \bar{w}$ aux personnes qui se trouvent en situation de chômage involontaire (2005, p. 118). Or, pour qu'un tel revenu minimal ne soit pas générateur d'inefficacité économique, il faut qu'il ne désincite pas au travail. Par conséquent, $k \bar{w}$ ne doit pas dépasser le plus bas revenu qu'obtient une personne en travaillant à temps plein $(2005$, p. 190). La redistribution est ainsi limitée de sorte que le coefficient $k$ soit choisi sur un intervalle allant non pas de 0 à 1 inclus, mais de 0 à $k_{e}$, tel que $k_{e} \bar{w}$ soit voisin du plus bas revenu obtenu par une personne travaillant à temps plein ${ }^{15}$ (2005, p. 285). De cette façon, tous les schémas ELIE intermédiaires qui souffraient du même type d'inefficacité que la structure ELIE ayant un coefficient de redistribution égal à 1, et relevaient ainsi d'une forme d' "esclavage des talentueux», sont écartés de la structure générale ELIE.

Kolm reconnaît d'ailleurs le fait qu'une redistribution « excessive », c'est-à-dire qui ne se contente pas d'effacer les différences entre capacités productives faibles et élevées, mais en vient à en inverser les positions devient une « situation d'exploitation des plus aptes » (2005, p. 116).

Le second point consiste à noter que l'argument que nous venons de qualifier de « philosophique » contre une redistribution totale des revenus ne s'applique pas aux schémas ELIE intermédiaires. En effet, dans la situation décrite par Dworkin, les personnes les plus productives sont obligées de travailler. Dans le modèle ELIE, le temps de travail est librement choisi - ce qui s'accorde avec sa volonté de placer la liberté sociale au fondement de cette construction.

«Les gens n'ont pas à payer pour leurs avantages donnés dont ils ne profitent pas, même s'ils choisissent volontairement cette abstention. Un tel impôt "violerait nos libertés de base" (Rawls, 2001). La macrojustice redistribue des avantages donnés, mais seulement parmi ceux de ces avantages dont le profit est effectivement tiré » (Kolm, 2007a, p. 79).

Cela amène Kolm à ne pas appliquer la règle générale de la structure ELIE aux personnes travaillant moins que $k$. Notons que la limite haute posée pour le coefficient $k$ de redistribution des revenus a de plus pour conséquence que le nombre de personnes tombant sous le coup de cette exception est très réduit (2007a, p. 79).

Le troisième point consiste, pour sa part, à montrer que l'argument économique qui s'opposait à la redistribution totale des revenus ne s'applique pas non plus aux schémas ELIE intermédiaires. Ces schémas ne désincitent pas les individus au travail mais, au contraire, les y incitent. En effet, puisque les individus révèlent leurs capacités productives par leur travail et la rémunération qui y est associée, réduire volontairement ses capacités productives ne serait pas du tout avantageux pour une personne qui a choisi de travailler plus que $k$. Minorer volontairement ses capacités productives reviendrait, en effet, à réduire volontairement son

15. Kolm emploie la formule « income-egalitarian equalization labour » pour désigner $k_{e}(2005$, p. 190). Le coefficient $k_{e}$ désigne le plus haut degré de redistribution avant que cette dernière ne devienne désincitative et donc génératrice d'inefficacité. L'égalisation maximale et, en même temps, efficace des revenus ne se confond donc pas avec $k=1$, mais avec $k=k_{e}$. 
revenu effectif. Dans les schémas intermédiaires, les individus restent donc incités à utiliser pleinement, et donc à révéler, leurs capacités productives s'ils travaillent au-delà de $k$. L'ensemble des $k$ éligibles étant borné supérieurement de sorte que le $k$ maximal soit voisin du plus bas revenu obtenu par une personne travaillant à temps plein, les schémas ELIE qui inciteraient à masquer ses capacités productives et même ceux qui n'inciteraient que faiblement à révéler lesdites capacités sont, par principe, exclus.

Un quatrième point peut encore être avancé pour appuyer l'idée que les schémas ELIE intermédiaires, à tout le moins ceux dont le coefficient $k$ est compris entre 0 et $k_{e}$, ne rentrent pas dans le cadre d'un " esclavage des talentueux »: la structure ELIE de redistribution des revenus doit être adoptée à l'unanimité. Et, malgré le caractère éminemment problématique de l'unanimité dans Macrojustice, force est de reconnaître qu'un tel cadre ne laisse aucune place à l'« esclavage » de qui que ce soit.

On doit donc admettre qu'il serait inapproprié de parler d' " esclavage des talentueux » dans le cas du modèle ELIE. De plus, il est important de noter que le refus de toute forme d' " esclavage des talentueux » conduit Kolm à n'appliquer la règle ELIE de redistribution qu'aux individus travaillant plus que $k$. Bien que cette "solution" pose à son tour d'autres problèmes (que nous n'aborderons pas ici), il est essentiel de prendre acte du fait que la représentation graphique du démembrement de la propriété de soi n'est donc pas le dernier mot de Kolm en termes de redistribution des revenus et qu'il n'entend pas du tout taxer le coût d'opportunité du loisir des individus.

Ce qui nous ramène à la question de savoir ce que Kolm entend par « libéralisme » et à celle de savoir en quoi sa position se distingue du libéralisme « classique ».

\subsection{Le sophisme du libéralisme classique}

La formule " libéralisme classique (classical process liberalism) 》 renvoie, dans Macrojustice, à toutes les positions qui font "l'éloge du marché sans politique de redistribution " (Kolm, 2005, p. 368). Cette définition extrêmement large du libéralisme classique permet à Kolm d'y inclure des penseurs aussi divers que Maurice Allais, Milton Friedman, Armen Alchian, John Hicks, Friedrich Hayek, James Buchanan, Murray Rothbard ou encore David Friedman (entre autres) - qu'ils aient placé explicitement ou pas la pleine propriété de soi au centre de leur réflexion (Kolm, 2005, p. 369).

Cette "théorie socio-éthique centrale du monde moderne » (Kolm, 2005, p. 20) se caractérise par l'importance fondamentale qu'elle accorde à la propriété et, en particulier, à la pleine propriété de soi qu'elle identifie à la liberté sociale (Kolm, 2005, p. 64). Toute atteinte à la pleine propriété de soi est alors forcément considérée comme une atteinte à la liberté sociale - ce qui justifie le refus de toute redistribution des revenus issus du travail.

Kolm montre que ce libéralisme classique commet un « sophisme majeur » (2006, p. 58) en identifiant la liberté sociale avec la pleine propriété de soi. En effet, l'identité entre deux termes suppose a minima leur équivalence, autrement dit leur implication réciproque. Il suffit donc de montrer qu'il n'y a pas d'implication réciproque entre pleine propriété de soi et liberté sociale pour prouver que ces deux concepts ne sont pas identiques.

C'est un fait que la pleine propriété de soi implique nécessairement la liberté sociale. En effet, si une personne est pleinement propriétaire d'elle-même, c'est-à-dire si elle possède les pleins usus, abusus et fructus d'elle-même, et donc de ses capacités productives, nul ne peut la contraindre à faire quelque chose qu'elle ne veut pas faire. En revanche, il est faux de dire que la liberté sociale implique nécessairement la pleine propriété de soi comme le prétend le libéralisme classique. Indiscutablement, si quelqu'un avait le droit de décider de l'usage que je dois faire d'une part de mes capacités productives, cela violerait ma liberté sociale. On doit 
admettre, par conséquent, que la liberté sociale implique le plein usus de soi-même et donc de ses propres capacités. Mais il ne va pas du tout de soi que la liberté sociale implique le plein fructus de celles-ci, comme le suppose le libéralisme classique. Rien ne contredit la liberté sociale dans le fait de devoir payer un droit de rente équivalent à la valeur d'une part de ses capacités productives. Et puisque la liberté sociale n'implique pas l'association des pleins usus, abusus et fructus de soi-même, il est faux de dire qu'elle implique la pleine propriété de soi.

Ce n'est donc pas une nécessité conceptuelle qui amène les tenants du libéralisme classique à refuser le fractionnement du fructus des capacités productives d'un individu. La prétendue identité entre la pleine propriété de soi et la liberté sociale est soit le résultat d'une erreur conceptuelle, soit une manière de masquer l'absence de nécessité d'un parti-pris théorique. Il n'y a, en effet, aucune contradiction à faire de la liberté sociale le principe fondamental de sa construction économique tout en refusant la pleine propriété de soi.

Nous nous trouvons ainsi en présence de deux grandes éthiques distributives dont l'une privilégie la pleine propriété de soi et s'oppose à toute forme de redistribution et l'autre privilégie la liberté sociale et pose la redistribution des revenus comme en découlant (Kolm, 2006, p. 71). Comment choisir entre ces deux éthiques ? Quel critère nous permettra de trancher en faveur de l'une ou l'autre de ces conceptions du libéralisme?

Kolm argue du fait que la règle générale de nos sociétés est la liberté et que cette valeur est le socle de nos constitutions (2005, p. 23). Il suffit de se référer aux textes fondateurs de nos systèmes politiques pour constater que la liberté y est posée en principe et que la propriété est présentée comme l'une de ses conséquences - et encore pourrait-on chercher en vain une mention explicite de la pleine propriété de soi. Toutefois, comme il va de soi qu'un tel argument, purement factuel, ne suffit pas à prouver la supériorité du libéralisme redistributif, Kolm y ajoute un argument conceptuel : chacun veut - tautologiquement et au nom de la justice - pouvoir faire ce qu'il veut faire, et par conséquent ne pas en être empêché par autrui. Chacun veut donc pour lui la liberté sociale. Or l'exigence de justice étant exigence d'impartialité, on doit admettre que ce que chacun juge juste et veut pour lui, doit être voulu pour tous (Kolm, 2006, p. 62).

Après avoir montré la non-identité de la liberté sociale et de la pleine propriété de soi, Kolm affirme donc le primat du principe de liberté sociale sur celui de propriété, ce qui lui permet de défendre un type de libéralisme non fondamentalement propriétariste ${ }^{16}$. Et c'est cette érection de la liberté sociale en principe premier de la société qui amène Kolm à baser la redistribution sur les capacités productives, inélastiques, plutôt que sur les revenus effectivement gagnés par les individus - et donc à prôner un impôt forfaitaire. Un impôt basé sur des variables dépendant des actions des individus ou de leurs conséquences (souhaitées) constituerait en effet pour ceux-ci une interférence contraignante et violerait par conséquent leur liberté sociale (Kolm, 2005, p. 66).

Dans la mesure où nous ne sommes pas pleinement et entièrement responsables de nos capacités productives individuelles, nous devons admettre que ces ressources nous sont, pour une partie, données - qu'elles sont en partie pour nous des ressources externes.

16. Nous disons bien «non fondamentalement propriétariste» et pas «fondamentalement non propriétariste ». Nous affirmons donc seulement que le libéralisme adopté par Kolm ne fait pas de la propriété, et en particulier de la pleine propriété de soi, son principe et son fondement. Il va de soi que Kolm donne un statut très important à la propriété et aux droits qui en sont constitutifs - dans la mesure où il s'attache justement à départager la part de nos capacités productives dont la propriété nous revient en droit. 
Deux auteurs d'importance utilisent la formule de « ressources externes »: Dworkin (1981, p. 307) et Van Parijs, (1995, p. 253, note 10). En qualifiant de " ressources externes » une partie des ressources que Dworkin et Van Parijs auraient appelés des « ressources internes », Kolm modifie profondément le sens que cette expression a chez ces deux auteurs puisqu'il s'agit d'une partie des capacités productives de l'individu. Tout cela découle du démembrement de la propriété de soi.

Le loyer qu'un individu paie sur cette part de ses capacités productives peut donc à bon droit être appelé « droits de rente externe » (Kolm, 2005, p. 61). Et puisqu'aucun individu ne peut revendiquer de propriété légitime et exclusive sur la part de capacités dont ce loyer est la valeur, on doit accepter (1) que ces ressources sont «données à la société 》 et peuvent donc être qualifiées de « ressources naturelles" (Kolm, 2006, p. 60) ; (2) qu'elles admettent un traitement normatif propre: qu'elles sont la propriété égale de tous et nécessitent, par conséquent, une politique de redistribution.

En pratique, la politique de redistribution sera évidemment le fait d'un Etat. Mais c'est à la société en tant que système de relations interindividuelles que les ressources naturelles sont données, puisque ce sont les autres individus qui sont « fruitiers partiels » de mes capacités productives. Dans Macrojustice, Kolm mentionne très régulièrement la société ou le social pour désigner la solidarité des individus et la communauté qu'ils constituent, mais il ne mentionne que très rarement l'Etat - qui semble n'avoir, dans son optique, de légitimité qu'en tant qu'émanation de la société.

Le libéralisme classique et celui que défend Kolm impliquent donc deux conceptions tout à fait différentes de la place de l'homme dans la société. La pleine propriété de soi, qui fonde le premier, fait de la société un système d'individus qui ne sont reliés que par des relations d'échanges et pouvant avoir de très grandes inégalités sur le plan économique. Le second voit, au contraire, la société comme un cadre de valeurs partagées par tous les individus et étant le lieu de transferts plus ou moins importants, résultants de la solidarité entre les personnes et de leur sens de la communauté. Dans cette société, chaque personne revendiquerait un droit sur une partie des capacités productives de chaque autre et se reconnaitrait le devoir de partager une part des fruits de ses aptitudes avec les autres.

\section{Kolm et les libertariens}

En partant de cette lecture du démembrement de la propriété de soi, il devient possible de comparer la position de Kolm à celles des libertariens de droite (4.1) et de gauche (4.2). Cela nous permettra de préciser le sens et les implications de cette théorie qui a été élaborée pour justifier la redistribution des revenus issus du travail, forme de redistribution à laquelle s'opposent par principe tous les libertariens.

\subsection{Le libertarisme de droite}

Dans les textes de Kolm, les mots « libertariens » et « libertarisme », font généralement référence au libertarisme de droite $(2007 \mathrm{~b}$, p. 37-38) - ils sont fréquemment associés aux noms de Murray Rothbard et de David Friedman (1996, p. 351 ; 2005, p. 252 et p. 369), dont l'appartenance à ce courant ne fait aucun doute ${ }^{17}$. Le libertarisme de droite ne fait pourtant

17. Le nom de Robert Nozick, qui peut à bon droit être qualifié de « libertarien de droite » (Vallentyne, 1999, p. 865) - puisqu'il défend la pleine propriété de soi et refuse, pour cette raison, toute redistribution des revenus (Nozick, 1974, p. 169 et p. 171) -, n'est pas explicitement rattaché au libertarisme dans Macrojustice. Toutefois, dans la mesure où le minarchisme nozickéen est considéré comme une variante du libéralisme classique - défini, justement, par ce refus de la redistribution au nom de la pleine propriété de soi -, cela ne pose aucune difficulté. 
pas l'objet d'un traitement spécifique, car il est replacé dans un courant plus large : le libéralisme classique, qui défend la pleine propriété de soi, s'oppose à toute forme de redistribution, et correspond donc bien, à ces égards tout au moins, à la position défendue par les libertariens de droite.

Il devient alors évident que la théorie de Kolm se construira en opposition au libertarisme de droite : puisque l'ampleur de la redistribution mesure le degré de communauté et de solidarité d'une société (2005, p. 285), le fait qu'une société choisisse un coefficient de redistribution nul mettrait en question le fait même que cette société en soit une - une telle société ne serait, en fait, qu'une juxtaposition d'individus sans rien de « social » qui les relie. Si le modèle ElIE n'exclut pas, par principe, qu'une société particulière choisisse de ne pas $\mathrm{du}$ tout redistribuer les revenus, Macrojustice est écrit pour prendre position contre le libéralisme classique, dont le libertarisme de droite est un éminent représentant. Dans cette optique, il est essentiel de comprendre que l'appel à la distinction opérée par Marx entre une liberté seulement « formelle » et une liberté « réelle » $(2005$, p. 24 et p. 57 ; 2006, p. 67) n'est pas seulement une référence convenue. Bien au contraire, il s'agit de l'un des aspects les plus fondamentaux du modèle ELIE de redistribution des revenus. La principale raison qui amène Kolm à interroger à nouveaux frais les fondements du libéralisme classique, et donc du libertarisme de droite, est, en effet, la constatation qu'ils ne garantissent qu'une liberté formelle quand l'idée de justice prescrit, selon lui, comme objectif d'une société qui fait de la liberté son principe, une liberté réelle minimale pour tous ses membres. C'est aussi de cette exigence de liberté réelle qui justifie la redistribution des revenus et donc le modèle ELIE.

Cet appel à la liberté réelle est donc le pivot qui conduit Kolm à se démarquer du libertarisme de droite. En remettant en cause l'identité de la liberté sociale et de la pleine propriété de soi, Kolm peut avancer l'idée du démembrement de cette dernière - et sans cela la redistribution des revenus issus du travail ne pourrait qu'être assimilable à du travail forcé (Nozick, 1974, p. 169) ou à une forme subtile et organisée de vol (Rothbard, 1973, p. 24).

Une fois que l'on a montré que la liberté n'implique pas analytiquement la pleine propriété de soi, et donc autorise son démembrement, le principal argument qu'il reste aux tenants du libéralisme classique, et donc aussi à ceux du libertarisme de droite, se résume à leur appel commun à l'efficacité du libre fonctionnement du marché. Toute théorie de l'imposition (corrélat nécessaire de la redistribution) en influant sur les mécanismes du marché court, en effet, le risque d'engendrer des effets de distorsion, et donc de l'inefficacité. Afin de se prémunir contre cette objection, Kolm choisit une base d'imposition inélastique (les capacités productives individuelles) - admettant qu'une théorie donnant par construction des résultats sous-optimaux serait disqualifiée par le fait même. L'inélasticité des capacités productives garantit ainsi que leur imposition ne rende pas le marché moins efficace qu'il ne l'est lorsqu'elles ne sont soumises à aucun prélèvement en vue de redistribution.

L'association des deux aspects essentiels du travail conceptuel de Kolm sur la nature de l'assiette de redistribution que sont l'inélasticité des capacités productives individuelles et le démembrement de la propriété de soi lui permettent donc de répondre à Nozick que nous pouvons ne pas être pleinement propriétaire de nous-mêmes sans que cela atteigne pour autant à notre liberté - que l'abandon de la pleine propriété de soi, corrélat de la redistribution des revenus issus du travail, ne suffit pas logiquement à nous condamner à une quelconque forme de travaux forcés.

\subsection{Le libertarisme de gauche}

Alors que la relation de Kolm au libertarisme de droite est une opposition assez franche, sa position vis-à-vis du libertarisme de gauche ne peut qu'être plus nuancée. Il partage, en 
effet, avec ce courant son refus d'une liberté qui pourrait n'être que formelle (Vallentyne, 1999, pp. 862-863) et il défend, pour cette raison, une redistribution des revenus - autrement dit, une forme d'égalisation de la liberté réelle des individus.

Vallentyne (1999, p. 860 et p. 872) avait d'ailleurs, avec beaucoup de justesse, noté une grande proximité thématique entre Le contrat social libéral (Kolm, 1985), qui consacrait tout un chapitre aux ressources naturelles et à leur distribution, et le libertarisme de gauche. Et le fait que Kolm considère les capacités productives individuelles, qui constituent la base de son assiette de redistribution, comme des ressources naturelles (Kolm, 2005, pp. 82-83) est encore un élément allant dans ce sens.

On aurait toutefois tort de vouloir conclure de cette proximité à l'idée que Kolm est un libertarien de gauche - aussi atypique soit-i1 ${ }^{18}$. En effet, dans leur réponse conjointe à la critique adressée par Fried (2004) au libertarisme de gauche, Vallentyne, Steiner \& Otsuka (2005, p. 201) définissent le libertarisme de gauche par deux principes : 1) la pleine propriété de soi ; 2) l'égale propriété des ressources naturelles. Dans la mesure où Kolm refuse ce premier principe, on doit reconnaître et insister sur le fait que sa position se distingue tout autant du libertarisme de droite que du libertarisme de gauche.

Si Kolm ne mentionne quasiment jamais nommément le libertarisme de gauche ${ }^{19}$, il se confronte indiscutablement à cette position lorsqu'il traite de la place que l'on doit accorder aux ressources naturelles non humaines dans la macrojustice. Il est en effet contraint de montrer que ces ressources ne sont pas une assiette de redistribution satisfaisante - sans quoi tout son travail de sape de la pleine propriété de soi, et son démembrement subséquent, perdrait à la fois, et leur nécessité pratique, et leur sens théorique.

Kolm avance trois raisons pour justifier son choix des capacités productives plutôt que des ressources naturelles non humaines comme principale assiette de la redistribution, raisons qui appuient l'idée que cette dernière solution est « impraticable » $(2005$, p. 88) :

- Premièrement, la faible importance relative de la redistribution que les ressources naturelles non humaines pourraient générer.

- Deuxièmement, le fait que l'allocation des ressources naturelles non humaines est souvent considérée comme un problème local et relevant, pour cette raison, du domaine de la microjustice.

- Troisièmement, celui que la plus grande partie des ressources naturelles non humaines appartiennent déjà à quelqu'un, et cela de longue date.

Ces trois arguments contre le choix des ressources naturelles non humaines en guise d'assiette de redistribution méritent d'être un peu creusés.

Le premier, malgré son apparence purement quantitative, est un argument strictement qualitatif. Kolm estime en effet que le travail, le capital et les ressources naturelles non humaines sont « responsables » respectivement de $80 \%, 18 \%$ et $2 \%$ de la valeur du revenu national. Cela lui permet de dire que, du fait que le capital a lui-même été produit précédemment, la part totale des ressources naturelles non humaines dans la production de valeur est de $2,5 \%^{20}$, et, par conséquent, la part au travail de 97,5\% (2005, p. 84). Si l'on

18. Wolfelsperger n'utilise l'expression de « libertarien de gauche non-orthodoxe » au sujet de Kolm que pour s'en démarquer et lui préférer celle d' « égalitariste parétien » (2008) - et cela justement pour la raison que nous invoquerons, à savoir le refus kolmien de la pleine propriété de soi.

19. Il n'y a, à ce jour, qu'une seule exception à cette règle : Kolm (2011c) mentionne explicitement le « libertarisme de gauche» en l'associant aux noms de Steiner et de Vallentyne (p. 353). Steiner et Vallentyne sont aussi associés, dans le même volume, à l'appellation « libéraux classiques de gauche » (2011b, p. 61).

20. Ce qui correspond aux $2 \%$ de la première estimation auxquels on ajoute la part que les ressources naturelles non humaines ont eu dans la production du capital, soit $18 \times$ x $/(80+2)]$. 
accorde ce point (qui pourrait sans doute être discuté), on doit reconnaître que la redistribution de la richesse basée sur les ressources naturelles non humaines est nécessairement insignifiante au regard de celle qui prendrait pour assiette le travail - comme le fait le modèle ELIE en taxant les capacités productives individuelles. L'objectif déclaré de Kolm est de garantir une liberté réelle «minimale». Malgré l'aspect indéterminé de ce qualificatif, cela revient à garantir à chaque membre de la société une liberté " suffisante » et il faut souligner que l'indétermination de ce terme n'est pas, en l'occurrence, un manque, mais la latitude laissée à chaque société de déterminer ce qu'elle entend par "une liberté réelle minimale suffisante », autrement dit de déterminer le coefficient $k$ de redistribution des revenus. Le rejet des ressources naturelles non humaines comme assiette de la redistribution des revenus tient ainsi essentiellement au fait que la liberté n'existe réellement qu'à partir d'un certain seuil en termes de quantité, ce qui explique qu'un argument quantitatif s'avère avoir un sens qualitatif.

Le second argument repose sur la distinction opérée par Kolm entre les trois niveaux de justice sociale que sont la macrojustice, la mésojustice et la microjustice (2005, pp. 15-16). La macrojustice traite des règles fondamentales de la société et de la distribution globale des revenus et des ressources, selon des critères généraux appliqués à tous, qui en découle. La mésojustice s'occupe de la répartition de biens dont la répartition n'est pas générale, mais qui pourtant revêtent une grande importance et concernent tout le monde (comme l'éducation ou la santé). Et la microjustice constitue le cadre de l'attribution à des individus particuliers de ressources trop rares pour pouvoir être données à tous et qui sont, par conséquent, attribuées en fonction de critères qui ne sont pas généraux (on pourra penser, entre autres, aux greffes d'organes). Or, il arrive souvent que les bénéfices tirés d'une ressource naturelle non humaine soient attribués exclusivement aux personnes qui vivent à l'endroit où elle se trouve, ce qui exclut cette ressource du champ de la redistribution générale, qui est celui de la macrojustice.

Le troisième argument se réfère à la légitimité de la propriété, que Kolm, nous l'avons souligné, ne prétend absolument pas remettre en cause en démembrant la propriété de soi. Même si l'on refuse le principe du premier occupant comme source légitime de la propriété, on doit accorder que la plus grande partie de ce que nous reconnaissons comme des ressources naturelles (terres, gisement de minerai, etc.) a été légitimement acquis par leurs propriétaires actuels et qu'il est impensable d'envisager une quelconque « rectification » de l'appropriation passée, car elle supposerait des expropriations incompatibles avec le respect de la propriété privée. Le nerf de cet argument tient dans le lien qui existe entre propriété privée et liberté sociale. On ne peut en effet porter atteinte à la première sans porter atteinte à la seconde puisque la liberté sociale se définit comme le fait que personne ne peut exercer de contrainte sur moi et que, pour moi, me trouver, hors de tout consentement de ma part, dépossédé de ma propriété serait indiscutablement une contrainte exercée de l'extérieur.

On remarquera que les trois arguments se combinent et qu'ils peuvent alors être compris comme une façon subtile de renverser le raisonnement des libertariens de gauche, dans la mesure où ils en viennent à opposer la propriété privée, qui, pour un libertarien, trouve son fondement dans la pleine propriété de soi, à une redistribution basée sur les ressources naturelles. La faiblesse du second principe des libertariens de gauche (l'égale propriété des ressources naturelles non humaines), qui se révèle incapable de justifier une redistribution " suffisante » des richesses, joue donc, selon Kolm, contre la pleine propriété de soi - et le démembrement de cette dernière apparaît comme une conséquence de l'exigence de liberté réelle minimale pour chaque membre d'une société qui se prétend « libérale ».

Il est important de noter que, malgré son opposition au libertarisme de gauche, les arguments de Kolm ne prétendent pas du tout exclure les ressources naturelles non humaines de la redistribution. Il leur accorde, tout au contraire, une place - auxiliaire. Les ressources 
naturelles non humaines sont insuffisantes pour financer la redistribution des revenus ${ }^{21}$, mais cela ne les en exclut pas pour autant.

Il pourrait sembler que cette déclaration s'oppose au troisième point de notre liste, à savoir au fait que la plus grande partie des ressources naturelles non humaines appartiennent à quelqu'un. Toutefois, ce qui est socialement reconnu comme relevant des ressources naturelles est susceptible de variation et de débats. De plus, des ressources naturelles peuvent être découvertes ou s'imposer alors qu'on ne leur prêtait aucun intérêt auparavant. Ainsi la mise en place d'un marché des permis d'émission de $\mathrm{CO}_{2}$ peut-elle être comprise comme la prise en considération de la qualité environnementale comme une ressource naturelle. Ces ressources naturelles encore non appropriées posent donc la question d'une éventuelle redistribution.

\section{Conclusion}

Pour achever de circonscrire l'apport de la théorie kolmienne du démembrement de la propriété de soi, il nous semble devoir faire trois brèves remarques conclusives.

La justification classique de la redistribution des revenus issus du travail s'inscrit dans la tradition welfariste et consiste à dire qu'un euro prélevé à une personne riche lui enlève moins d'utilité qu'il n'en apporte au pauvre qui le reçoit. La théorie du démembrement de la propriété de soi fournit pour sa part une justification libérale à la redistribution des revenus qui ne se réfère pas à l'idée d'une maximisation de l'utilité collective et qui affronte directement la question de la perte de liberté impliquée par les prélèvements nécessaires à toute redistribution. Les autres approches défendues par les égalitaristes libéraux arguaient soit de la propriété collective des capacités individuelles, soit de l'obligation (d'ordre moral) que la société garantisse aux individus une liberté réelle minimale. La théorie du démembrement de la propriété de soi, qui tente d'un même mouvement de creuser ces approches et de leur apporter un fondement, ne prétend donc pas seulement être une justification libérale possible de la redistribution des revenus issus du travail, mais bien la justification libérale de ce type de redistribution - partagée de manière implicite par toutes positions appartenant à l'« égalitarisme libéral».

Il est extrêmement important de noter le rôle accordé à la philosophie dans Macrojustice. Le modèle ELIE n'est pas seulement fondé sur une réflexion philosophique préalable et qui lui resterait ainsi extérieure. Il est le fruit d'une démarche dans laquelle philosophie et économie se complètent et s'imbriquent tout au long de la réflexion. La dialectique entre arguments philosophiques et arguments économiques est tellement serrée qu'il faut bien reconnaître que les distinguer revient à faire une distinction de pure raison à l'intérieur d'un continuum argumentatif. «L'association de l'économie et de la philosophie [...] n'est pas seulement un aspect louable (et probablement trop rare) dans la recherche académique (scholarship); dans une branche telle que l'économie normative, elle est simplement inévitable pour que ce que l'on énonce ait un sens et pour progresser (en fait, je pense que l'opinion correcte consiste à ne faire aucune différence entre les deux champs) » (Kolm, 2011a, p. 38).

La théorie du démembrement de la propriété de soi a indiscutablement une dimension rhétorique. Affirmer cela, ce n'est toutefois pas dire que la théorie elle-même est rhétorique (car en tant que fondement de la théorie kolmienne de la propriété, elle a une portée qui ne saurait être réduite à cela), c'est seulement relever que le choix de l'expression

21. Rappelons que le niveau actuel de redistribution dans les pays les plus industrialisés se situe selon Kolm entre 20 et $40 \%$ de la richesse produite (Kolm, 2007a, p. 78). 
« démembrement de la propriété de soi » souligne l'opposition du libéralisme défendu par Kolm avec le libéralisme classique (et par conséquent, avec toutes les formes de libertarisme).

\section{Bibliographie}

Cohen G. A. (1995), Self-Ownership, Freedom and Equality, Cambridge, Cambridge University Press.

Christman J. (1991), "Self-Ownership, Equality, and the Structure of Property Rights », Political Theory, vol. 19/1, pp. 28-46.

de la Croix D. \& Lubrano M. (2011), « The Trade-off Between Growth and Redistribution: ELIE in an Overlapping Generations Model », in Gamel C. \& Lubrano M. (ed.) (2011), pp. 305-337.

Dworkin R. (1981), « What Is Equality? » Part I : « Equality of Welfare » ; Part II : « Equality of Resources », Philosophy \& Public Affairs, vol. 10/3, pp. 185-245 et vol. 10/4, pp. 283-345.

Fleurbaey M. \& Maniquet F. (2011), « Kolm's Tax, Tax Credit, and the Flat Tax », in Fleurbaey M., Salles M. \& Weymark J. (ed.) (2011), pp. 217-239.

Fleurbaey M., Salles M. \& Weymark J. (ed.) (2011), Social Ethics and Normative Economics, Essays in Honour of Serge-Christophe Kolm, Berlin, Heidelberg, New-York, Springer Verlag.

Fried B. (2004), « Left-Libertarianism: A Review Essay », Philosophy \& Public Affairs, vol. 32/1, pp. 67-92.

Gamel C. (2005), «Recension d'ouvrage : "Serge-Christophe Kolm, Macrojustice - The Political Economy of Fairness" ", Revue de philosophie économique, $\mathrm{n}^{\circ}$ 12, pp. 181-191.

Gamel C. \& Lubrano M. (ed.) (2011a), On Kolm's Theory of Macrojustice : A Pluridisciplinary Forum of Exchange, Berlin, Heidelberg, New-York, Springer Verlag.

Gamel C. \& Lubrano M. (2011b), « Why Should We Debate the Theory of Macrojustice? », in Gamel C. \& Lubrano M. (ed.) (2011a), pp. 1-32.

Gharbi J.-S \& Sambuc C. (2012), «Propriété de soi et justice sociale chez les libertariens », Cahiers d'économie politique, $\mathrm{n}^{\circ} 62$, pp. 187-222.

Gharbi J.-S. \& Meinard Y. (2015), «On the Meaning of Non-Welfarism in Kolm's ELIE Model of Income Redistribution », Journal of Economic Methodology, à paraître.

Gorr M. (1995), «Justice, Self-Ownership, and Natural Assets », Social Philosophy and Policy, vol. 12/2, pp. 267-271.

Kernohan A. (1990), « Rawls and the Collective Ownership of Natural Abilities », Canadian Journal of Philosophy, vol. 20/1, pp. 19-28.

Kolm S.-C. (1985), Le contrat social libéral, Philosophie et pratique du libéralisme, Paris, PuF.

Kolm S.-C. (1996), Modern Theories of Justice, Cambridge (MA), Mit Press.

Kolm S.-C. (2005), Macrojustice - The Political Economy of Fairness, Cambridge, Cambridge University Press.

Kolm S.-C. (2006), « Liberté, justice et efficacité : distribution, impôts et transferts optimaux », Revue économique, vol. 57/1, pp. 55-84.

Kolm S.-C. (2007a), " Macrojustice : Distribution, transferts et impôts optimaux », Revue d'économie politique, vol. 117/1, pp. 61-89.

Kolm S.-C. (2007b), «Qu'est-ce qu'un impôt juste?», Regards Croisés sur l'Economie, n 1, pp. 37-50. 
Kolm S.-C. (2009), « La défiscalisation des heures supplémentaires : la théorie », Raisons politiques, $\mathrm{n}^{\circ} 33$, pp. $45-55$.

Kolm S.-C. (2011a), «General Presentation », in Gamel C. \& Lubrano M. (éd.) (2011a), pp. 35-67.

Kolm S.-C. (2011b), « Economic Macrojustice: Fair Optimum Income Distribution, Taxation and Transfers », in Gamel C. \& Lubrano M. (ed.) (2011a), pp. 69-130.

Kolm S.-C. (2011c), « Macrojustice in Normative Economics and Social Ethics », in Gamel C. \& Lubrano M. (ed.) (2011a), pp. 341-370.

Lubrano M. (2011), «The Redistributive Aspects of ELIE: A Simulation Approach », in Gamel C. \& Lubrano M. (ed.) (2011a), pp. 275-304.

Locke J. (1690), Second Treatise of Governement ; traduction française : Second traité du gouvernement, Paris, PUF, (1994) (édition citée).

Nozick R. (1974), Anarchy, State and Utopia, Oxford, Basil Blackwell.

Ooghe E. \& Schokkaert E. (2011), « Is ELIE a Wasteful Minimum Income Scheme? », in Gamel C. \& Lubrano M. (ed.) (2011a), pp. 235-255.

Overton R. (1646), An Arrow Against All Tyrants ; réédition in Aylmer G. E. (éd.), The Levelers in the English Revolution, London, Thames and Hudson (1975).

Rawls J. (1971), A Theory of Justice, Cambridge. MA. Harvard University Press ; traduction française : Théorie de la justice, Paris, Le seuil, (1997) (édition citée).

Rothbard M. (1973), For a New Liberty, the Libertarian Manifesto, New York: Libertarian Review Foundation.

Sen A. K. (1979), «Utilitarianism and Welfarism », Journal of Philosophy, vol. 76/9, pp. 463-489.

Sen A. K. (1980), «Equality of What? », in McMurrin S. (ed.) The Tanner Lectures on Human Values, vol. 1, University of Utah Press, pp. 197-220 ; traduction française : «Quelle égalité ?» in Sen A. K. (1993), Ethique et économie, Paris, PUF, pp. 189-213.

Simula L. \& Trannoy A. (2011a), «When Kolm Meets Mirrlees: ELIE », in Fleurbaey, M., Salles M. \& Weymark J. (ed.) (2011), p. 193-216.

Simula L. \& Trannoy A. (2011b), « An Exploration of Incentive-Compatible ELIE », in Gamel C. \& Lubrano M. (ed.) (2011a), pp. 207-231.

Taylor R. S. (2005), « Self-Ownership and the Limits of Libertarianism », Social Theory and Practice, vol. $31 / 4$, pp. $465-482$.

Vallentyne P. (1999), «Le libertarisme de gauche et la justice», Revue économique, vol. 50/4, pp. 859-878.

Vallentyne P., Steiner H. \& Otsuka M. (2005), «Why Left-Libertarianism is not Incoherent, Indeterminate, or Irrelevant: A Reply to Fried», Philosophy \& Public Affairs, vol. 33/2, pp. 201-215.

Van Parijs P. (1995), Real Freedom For All, What (If Anything) Can Justify Capitalism ?, Oxford, Oxford University Press.

Wolfelsperger A. (2008), "The Philosophical Foundations of Kolm's Theory of Macrojustice », miméo. 